\title{
QUASI-CONFORMAL CURVATURE TENSOR ON SASAKIAN MANIFOLDS
}

\section{Riddhi Jung Shah, N. V. C. Shukla}

Journal of Institute of Science and Technology

Volume 22, Issue 1, July 2017

ISSN: 2469-9062 (print), 2467-9240 (e)

\section{Editors:}

Prof. Dr. Kumar Sapkota

Prof. Dr. Armila Rajbhandari

Assoc. Prof. Dr. Gopi Chandra Kaphle

JIST, 22 (1): 94-98 (2017)

\section{Published by:}

Institute of Science and Technology

Tribhuvan University

Kirtipur, Kathmandu, Nepal 


\title{
QUASI-CONFORMAL CURVATURE TENSOR ON SASAKIAN MANIFOLDS
}

\author{
Riddhi Jung Shah ${ }^{1 *}$, N. V. C. Shukla ${ }^{2}$ \\ ${ }^{1}$ Department of Mathematics, Janata Campus, Nepal Sanskrit University, Dang, Nepal \\ ${ }^{2}$ Department of Mathematics \& Astronomy, University of Lucknow, Lucknow-226007, India \\ *Corresponding E-mail: shahrjgeo@gmail.com
}

\begin{abstract}
In this paper we studied some curvature properties of quasi-conformal curvature tensor on Sasakian manifolds. We have proven that a $(2 n+1)$-dimensional Sasakian manifold satisfying the curvature conditions $R(X, Y) . S=0$ and $R(X, Y) \cdot W=0$ is an Einstein manifold. We have also obtained some results on quasi-conformally recurrent Sasakian manifold. Finally, Sasakian manifold satisfying the condition $\operatorname{div} W=0$ was studied.
\end{abstract}

Keywords: Sasakian manifold, Quasi-conformal curvature tensor, Einstein manifold, Ricci semi-symmetric, Quasi-conformally recurrent.

\section{INTRODUCTION}

The notion of Sasakian structure was introduced by Sasaki (Sasaki, 1960). The geometry of the Sasakian manifolds is very important as it is widely used in mathematical physics. Sasakian manifolds have been studied by many and some of them are Boyer \& Galicki (2008), Godlinski et al. (2000), Shah (2013). On the other hand, quasi-conformal curvature tensor was defined and studied by Yano \& Sawaki (1968). This curvature tensor has also been studied by Adati \& Miyazawa (1967), Amur \& Maralabhavi (1977) and many others.

\section{PRELIMINARIES}

If the contact metric structure $(\varphi, \xi, \eta, g)$ of a differentiable manifold $M$ is normal then the structure is called a Sasakian structure or a normal contact metric structure, where $\varphi$ is a $(1,1)$ tensor field, $\xi$ is a Reeb vector field, $\eta$ is a 1 -form and $g$ is a compatible Riemannian metric. A $(2 n+1)$ dimensional differentiable manifold $M$ equipped with Sasakian structure is called a Sasakian manifold.

In a $(2 n+1)$-dimensional Sasakian manifold we have the following fundamental relations

$\left\{\begin{array}{l}\varphi^{2}(X)=-X+\eta(X) \xi, \varphi \xi=0, \\ \eta(\varphi X)=0, \eta(\xi)=1\end{array}\right.$

$$
\begin{aligned}
& \left\{\begin{array}{l}
g(X, \xi)=\eta(X), \\
g(\varphi X, \varphi Y)=g(X, Y)-\eta(X) \eta(Y)
\end{array}\right. \\
& \left(\nabla_{X} \varphi\right) Y=g(X, Y) \xi-\eta(Y) X \\
& \nabla_{X} \xi=-\varphi X \\
& \left(\nabla_{X} \eta\right)(Y)=g\left(\nabla_{X} \xi, Y\right)=-g(\varphi X, Y)
\end{aligned}
$$

for all $X, Y \in T M$, where $\nabla$ is the Levi-Civita connection of Riemannian metric $g$ (Blair, 1976). The fundamental 2-form $\Phi$ in normal structure is defined as:

$$
\begin{array}{r}
\Phi(X, Y)=d \eta(X, Y)=g(X, \varphi Y) \\
=-g(X, \varphi Y)
\end{array}
$$

In a $(2 n+1)$-dimensional Sasakian manifold we also have

$$
\begin{aligned}
& \eta(R(X, Y) Z)=g(Y, Z) \eta(X)-g(X, Z) \eta(Y) \\
& R(X, Y) \xi=\eta(Y) X-\eta(X) Y \\
& R(\xi, X) Y=g(X, Y) \xi-\eta(Y) X \\
& Q \varphi=\varphi Q
\end{aligned}
$$




$$
\begin{aligned}
& S(X, \xi)=2 n \eta(X) \\
& S(\varphi X, Y)=-S(X, \varphi Y) \\
& S(\varphi X, \varphi Y)=S(X, Y)-2 n \eta(X) \eta(Y)
\end{aligned}
$$

where, $R, S$ and $Q$ are the curvature tensor, the Ricci tensor and the Ricci operator respectively.

In a $(2 n+1)$-dimensional Sasakian manifold the quasi-conformal curvature tensor $W(X, Y) Z$ of type $(1,3)$ is defined as:

$$
\begin{aligned}
W & (X, Y) Z \\
= & a R(X, Y) Z+b[S(Y, Z) X-S(X, Z) Y] \\
& +g(Y, Z) Q X-g(X, Z) Q Y] \\
& -\frac{r}{(2 n+1)}\left\{\frac{a}{2 n}+2 b\right\}\{g(Y, Z) X \\
& -g(X, Z) Y\}
\end{aligned}
$$

where $a, b$ are arbitrary constants such that $a, b \neq 0$ and $r$ is the scalar curvature.

Definition 1: A $(2 n+1)$-dimensional Sasakian manifold $M$ is said to be Ricci-semisymmetric if it satisfies the condition

$$
R(X, Y) \cdot S=0
$$

Where, $R(X, Y)$ is the curvature operator and $S$ is the Ricci tensor of type $(0,2)$ (Szabo 1982).

Definition 2: A $(2 n+1)$-dimensional Sasakian manifold $M$ is said to be quasi-conformal semisymmetric if the condition

$$
R(X, Y) \cdot W=0
$$

holds for all $X, Y$ (Shaikh \& Biswas, 2004).

Definition 3: A $(2 n+1)$-dimensional Sasakian manifold $M$ is said to be an Einstein manifold if the Ricci tensor $S$ of type $(0,2)$ is of the form

$$
S(X, Y)=\lambda g(X, Y)
$$

where $\lambda$ is a scalar (Yano \& Kon 1984).

Definition 4: A non-flat Riemannian manifold $M$ is said to be quasi-conformally recurrent if the quasi-conformal curvature tensor $W$ satisfies the condition

$$
\nabla W=\alpha \otimes W
$$

where $\alpha$ is an everywhere non-zero 1-form (Yano \& Sawaki, 1968).

\section{RESULTS AND DISCUSSION}

We prove the following results:

Theorem 1: A $(2 n+1)$-dimensional Sasakian manifold satisfying the condition $R(X, Y) . S=0$ is an Einstein manifold and its scalar curvature tensor is $r=2 n(2 n+1)$.

Proof: Let $M$ be a $(2 n+1)$-dimensional Sasakian manifold. Suppose the Ricci semi-symmetric condition i.e., $(R(X, Y) . S)(U, V)=0$ holds in $M$, then by definition we have

$$
S(R(X, Y) U, V)+S(U, R(X, Y) V)=0 .
$$

Putting $V=\xi$ in (19) and using (8) and (11) we get

$$
\begin{aligned}
0= & 2 n \eta(R(X, Y) U)+\eta(Y) S(U, X) \\
& -\eta(X) S(U, Y) .
\end{aligned}
$$

Replacing $X$ by $\xi$ in (20) and using (1), (7), (9) and (11) we obtain

$$
S(U, Y)=2 n g(U, Y) \text {. }
$$

Equation (21) implies that the manifold $M$ is an Einstein manifold.

Let $\left\{e_{i}: i=1,2, \ldots, 2 n+1\right\}$ be an orthonormal basis of the tangent space at any point of the manifold. Putting $U=Y=e_{i}$ in (21) and taking summation over $i, 1 \leq i \leq 2 n+1$, we get

$r=2 n(2 n+1)$.

In view of (21) and (22), the theorem is proved.

Theorem 2: If a $(2 n+1)$-dimensional Sasakian manifold $M$ satisfies the quasi-conformal semisymmetric condition $(R(X, Y) . W)(U, V) Z=0$, then $M$ is an Einstein manifold.

Proof: Let us consider a $(2 n+1)$-dimensional Sasakian manifold $M$ which satisfies the condition $(R(X, Y) \cdot W)(U, V) Z=0$. Then, by definition we have

$$
\begin{aligned}
0 & =R(X, Y) W(U, V) Z-W(R(X, Y) U, V) Z \\
& -W(U, R(X, Y) V) Z-W(U, V) R(X, Y) Z .
\end{aligned}
$$


Taking $X=\xi$ in (23) and using (9) we get

$$
\begin{aligned}
0 & =g(W(U, V) Z, Y) \xi-\eta(W(U, V) Z) Y \\
& -g(Y, U) W(\xi, V) Z+\eta(U) W(Y, V) Z \\
& -g(Y, V) W(U, \xi) Z+\eta(V) W(U, Y) Z \\
& -g(Y, Z) W(U, V) \xi+\eta(Z) W(U, V) Y .
\end{aligned}
$$

Taking inner product on both sides of (24) by $\xi$ and using (1) and (2) we obtain

$$
\begin{aligned}
& g(W(U, V) Z, Y)-\eta(W(U, V) Z) \eta(Y) \\
& -g(Y, U) \eta(W(\xi, V) Z)+\eta(U) \eta(W(Y, V) Z) \\
& -g(Y, V) \eta(W(U, \xi) Z)+\eta(V) \eta(W(U, Y) Z) \\
& -g(Y, Z) \eta(W(U, V) \xi)+\eta(Z) \eta(W(U, V) Y) \\
& =0 .
\end{aligned}
$$

Now, in view of (14) and (22) we have

$$
\begin{aligned}
& \eta(W(U, V) Z) \\
& =b[S(V, Z) \eta(U)-S(U, Z) \eta(V) \\
& -2 n\{g(V, Z) \eta(U)-g(U, Z) \eta(V)\}] . \\
& \eta(W(U, V) \xi)=0 \\
& \eta(W(\xi, V) Z)=b[S(V, Z)-2 n g(V, Z)] \\
& \eta(W(U, \xi) Z)=b[2 n g(U, Z)-S(U, Z)] .
\end{aligned}
$$

Putting $Y=U$ in (25) and using (26)-(29) we obtain

$$
\begin{aligned}
& g(W(U, V) Z, U)-g(U, U) \eta(W(\xi, V) Z) \\
& -g(U, V) \eta(W(U, \xi) Z)+\eta(V) \eta(W(U, U) \xi) \\
& -g(U, Z) \eta(W(U, V) \xi)+\eta(Z) \eta(W(U, V) U)=0 \\
& \quad g(W(U, V) Z, U)-g(U, U)[b S(V, Z) \\
& \quad-2 n b g(V, Z)]-g(U, V)[2 n b g(U, Z) \\
& \text { or, }-b S(U, Z)]+\eta(Z)[b S(U, V) \eta(U) \\
& \quad-b S(U, U) \eta(V)-2 n b g(U, V) \eta(U) \\
& \quad+2 n b g(U, U) \eta(V)]=0
\end{aligned}
$$

$$
\begin{aligned}
& g(W(U, V) Z, U)-b g(U, U) S(V, Z) \\
& +2 n b g(U, U) g(V, Z) \\
& -2 n b g(U, V) g(U, Z) \\
& +b S(U, Z) g(U, V) \\
\text { or, } & +b S(U, V) \eta(U) \eta(Z) \\
& -b S(U, U) \eta(V) \eta(Z) \\
& -2 n b g(U, V) \eta(U) \eta(Z) \\
& +2 n b g(U, U) \eta(V) \eta(Z)=0 .
\end{aligned}
$$

Let $\left\{e_{i}: i=1,2, \ldots, 2 n+1\right\}$ be an orthonormal basis of the tangent space at any point of the manifold. Taking $U=e_{i}$ in (30) and summing over $i$, $1 \leq i \leq 2 n+1$, we get

$$
\begin{aligned}
(a-b) S(V, Z) & =\left[\left(4 n^{2}-r\right) b+2 n a\right] g(V, Z) \\
& +[\{r-2 n(2 n+1)\} b] \eta(V) \eta(Z) .
\end{aligned}
$$

From (31) we have

$$
\begin{aligned}
S(V, Z) & =\frac{1}{a-b}\left[\left(4 n^{2}-r\right) b+2 n a\right] g(V, Z) \\
& +\frac{1}{a-b}[\{r-2 n(2 n+1)\} b] \eta(V) \eta(Z)
\end{aligned}
$$

provided that $a-b \neq 0$. The relation (32) implies that the manifold is $\eta$-Einstein.

If $a=b$, then taking an orthonormal frame field and contracting over $V$ and $Z$,(31) yields

$$
r=2 n(2 n+1) \text {. }
$$

Using (33) in (32) we get

$$
S(V, Z)=2 n g(V, Z)
$$

provided $a-b \neq 0$. Thus the manifold is an Einstein manifold. This completes the proof of the theorem.

Theorem 3: If a $(2 n+1)$-dimensional Sasakian manifold $M$ is quasi-conformally recurrent, then it is an Einstein manifold and its scalar curvature is $r=2 n(2 n+1)$.

Proof: Let us consider a $(2 n+1)$-dimensional Sasakian manifold $M$ which is quasi-conformally recurrent. Then from (18) we have

$$
\left(\nabla_{U} W\right)(X, Y) Z=\alpha(U) W(X, Y) Z
$$

for some 1-form $\alpha$. 
Contracting (35) at $U$ we have

$(\operatorname{div} W)(X, Y) Z=g(W(X, Y) Z, P)$

where $P$ is a vector field metrically associated to the recurrent form $\alpha$.

Taking inner product on both sides of (14) by $V$ and differentiating covariantly along $U$ we obtain

$$
\begin{aligned}
& g\left(\left(\nabla_{U} W\right)(X, Y) Z, V\right) \\
& =a g\left(\left(\nabla_{U} R\right)(X, Y) Z, V\right) \\
& +b\left\{\left(\nabla_{U} S\right)(Y, Z) g(X, V)\right. \\
& -\left(\nabla_{U} S\right)(X, Z) g(Y, V) \\
& +\left(\nabla_{U} S\right)(X, V) g(Y, Z) \\
& \left.-\left(\nabla_{U} S\right)(Y, V) g(X, Z)\right\} \\
& -\frac{(a+4 n b)}{2 n(2 n+1)}\{g(Y, Z) g(X, V) d r(U) \\
& -g(X, Z) g(Y, V) d r(U)\} .
\end{aligned}
$$

Putting $U=V=e_{i}$ and taking summation over $i$, $1 \leq i \leq 2 n+1$, we get

$$
\begin{aligned}
& (\operatorname{div} W)(X, Y) Z \\
& =(a+b)\left\{\left(\nabla_{X} S\right)(Y, Z)-\left(\nabla_{Y} S\right)(X, Z)\right\} \\
& -\frac{a+n(3-2 n) b}{2 n(2 n+1)}\{d r(X) g(Y, Z) \\
& -d r(Y) g(X, Z)\} .
\end{aligned}
$$

Substituting (36) in (38) and taking $X=\xi$, we obtain

$$
\begin{aligned}
& (a+b)\left\{\left(\nabla_{\xi} S\right)(Y, Z)-\left(\nabla_{Y} S\right)(\xi, Z)\right\} \\
& -\frac{a+n(3-2 n) b}{2 n(2 n+1)}\{g(Y, Z) d r(\xi) \\
& -\eta(Z) d r(Y)\}-g(W(\xi, Y) Z, P)=0 .
\end{aligned}
$$

We also have

$$
\begin{aligned}
& \left(\nabla_{\xi} S\right)(Y, Z) \\
& =\left(L_{\xi} S\right)(Y, Z)-S\left(\nabla_{Y} \xi, Z\right)-S\left(Y, \nabla_{Z} \xi\right) .
\end{aligned}
$$

Since $\xi$ is a Killing vector field, we have $\left(L_{\xi} S\right)(Y, Z)=0$ and $d r(\xi)=0$, where $L$ is a Lie differentiation operator. By the use of (4) and (12) we obtain $S\left(\nabla_{Y} \xi, Z\right)+S\left(Y, \nabla_{Z} \xi\right)=0$. Thus above equation reduces to

$$
\left(\nabla_{\xi} S\right)(Y, Z)=0 .
$$

Again, we have

$$
\left(\nabla_{Y} S\right)(\xi, Z)=S(\varphi Y, Z)-2 n g(\varphi Y, Z) .
$$

In view of (9), (14), (39), (40) and (41), we obtain

$$
\begin{aligned}
& \frac{a+n(3-2 n)}{2 n(2 n+1)} \eta(Z) d r(Y)-(a+b)\{S(\varphi Y, Z) \\
& -2 n g(\varphi Y, Z)\} \\
& =\frac{2 n a(2 n+1)-(a+4 n b) r}{2 n(2 n+1)}\{g(Y, Z) \eta(P) \\
& -\eta(Z) g(Y, P)\}+b\{S(Y, Z) \eta(P) \\
& -2 n g(Y, P) \eta(Z)+2 n g(Y, Z) \eta(P) \\
& -S(Y, P) \eta(Z)\} .
\end{aligned}
$$

Replacing $Z$ by $\varphi Z$ in (42) and using (1), (2) and (13) we get

$$
\begin{aligned}
- & (a-b)\{S(Y, Z)--2 n g(Y, Z)\} \\
= & \eta(P)\left[\left\{\frac{a(2 n(2 n+1)-r)+2 n(2 n-2 r+1)}{2 n(2 n+1)}\right\}\right. \\
& \times g(Y, \varphi Z)+b S(Y, \varphi Z)] .
\end{aligned}
$$

Interchanging $Y$ and $Z$ in (43) and using (6) and (12) we obtain

$$
\begin{aligned}
& (a+b)\{S(Y, Z)-2 n g(Y, Z)\} \\
& =\eta(P)\left[\left\{\frac{a(2 n(2 n+1)-r)+2 n(2 n-2 r+1) b}{2 n(2 n+1)}\right\}\right. \\
& \quad \times g(Y, \varphi Z)+b S(Y, \varphi Z)] .
\end{aligned}
$$

Subtraction of (43) from (44) yields

$$
S(Y, Z)=2 n g(Y, Z)
$$

provided $a+b \neq 0$. Hence the manifold is Einstein. Taking an orthonormal frame field and contracting over $Y$ and $Z$ in (45), it follows that

$$
r=2 n(2 n+1)
$$

where $r$ is the scalar curvature of the Sasakian manifold. In view of (45) and (46), the theorem is proved.

Theorem 4: Let $M$ be a $(2 n+1)$-dimensional Sasakian manifold. If $M$ satisfies the condition $\operatorname{div} W=0$, then the manifold is an Einstein manifold. 
Proof: Let us consider a $(2 n+1)$-dimensional Sasakian manifold $M$ which satisfies the condition $\operatorname{div} W=0$, where ' $d i v W$ ' denotes the divergence of the quasi-conformal curvature tensor $W$. Then from (14) we obtain

$$
\begin{aligned}
& \left(\nabla_{V} W\right)(X, Y) Z \\
& =a\left(\nabla_{V} R\right)(X, Y) Z+b\left\{\left(\nabla_{v} S\right)(Y, Z) X\right. \\
& -\left(\nabla_{v} S\right)(X, Z) Y+g(Y, Z)\left(\nabla_{v} Q\right) X \\
& \left.-g(X, Z)\left(\nabla_{v} Q\right) Y\right\}-\frac{(a+4 n b) d r(V)}{2 n(2 n+1)} \\
& \times\{g(Y, Z) d r(X)-g(X, Z) d r(Y)\} .
\end{aligned}
$$

Contraction of (47) yields

$$
\begin{aligned}
& (\operatorname{div} W)(X, Y) Z \\
= & (a+b)\left\{\left(\nabla_{X} S\right)(Y, Z)-\left(\nabla_{Y} S\right)(X, Z)\right\} \\
& -\frac{a+n(3-2 n) b}{2 n(2 n+1)}\{g(Y, Z) d r(X) \\
& -g(X, Z) d r(Y)\} .
\end{aligned}
$$

For $\operatorname{div} W=0,(48)$ reduces to

$$
\begin{aligned}
& (a+b)\left\{\left(\nabla_{X} S\right)(Y, Z)-\left(\nabla_{Y} S\right)(X, Z)\right\} \\
& =\frac{a+n(3-2 n) b}{2 n(2 n+1)}\{g(Y, Z) d r(X) \\
& -g(X, Z) d r(Y)\} .
\end{aligned}
$$

Putting $X=\xi$ in (49) and proceeding as in the proof of theorem 3 we have $L_{\xi} S=0, d r(\xi)=0$ and consequently $\nabla_{\xi} S=0$. Therefore (49) yields

$$
\begin{aligned}
& (a+b)\{S(\varphi Y, Z)-2 n g(\varphi Y, Z)\} \\
& =\frac{a+n(3-2 n) b}{2 n(2 n+1)} \eta(Z) d r(Y) .
\end{aligned}
$$

Replacing $Z$ by $\varphi Z$ in (50) and using (1), (2) and (13) we obtain

$$
S(Y, Z)=2 n g(Y, Z) \text {, provided } a+b \neq 0 .
$$

This implies that the manifold is Einstein. This completes the proof of the theorem.

\section{CONCLUSION}

A $(2 n+1)$-dimensional Sasakian manifold satisfying the curvature conditions $R(X, Y) . S=0$ and $R(X, Y) \cdot W=0$ is an Einstein manifold. It is also proved that a Sasakian manifold has scalar curvature $r=2 n(2 n+1)$. We have obtained the result that quasiconformally recurrent Sasakian manifold is an Einstein manifold. This paper is useful for researchers who are working in the field of physics and structures on differentiable manifolds.

\section{ACKNOWLEDGEMENT}

The authors are thankful to the worthy referee for his/her useful suggestions for the improvement of the paper.

\section{REFERENCES}

Adati, T. and Miyazawa, T. (1967). On a Riemannian space with recurrent conformal curvature. Tensor N. S., 18: 348-354.

Amul, K. and Maralabhavi, Y. B. (1977). On quasiconformally flat spaces. Tensor N. S., 31: 194-198.

Blair, D. E. (1976). Contact manifolds in Riemannian geometry. Lecture Notes Mathematics, 509, Springer-Verlag, Berlin.

Boyer, C. P. Galicki, K. (2008). Sasakian geometry. Oxford Mathematical Monograph, Oxford University Press, Oxford.

Godlinski, M.; Kopezynski, W. and Nurowski, P. (2000). Locally Sasakian manifolds. Classical and Quantum Gravity, 17: 105-115.

Sasaki, S. (1960). On differentiable manifolds with certain structures which are closely related to almost contact structure. Tohoku Math Journal, 12: 459-476.

Shah, R. J. (2013). Properties of some curvature tensors on Sasakian manifolds. Journal of Institute of Science and Technology, 18 (1): 39-42.

Shaikh, A. A. and Biswas, S. (2004). On LPSasakian manifolds. Bulletin of Malaysian Mathematical Sciences Society, 27: 17-26.

Szabo, Z. I. (1982). Structure theorems on. Riemannian spaces satisfying $R(X, Y) \cdot R=0$, I, The local version. Journal of Differential Geometry, 17: 531-582.

Yano, K. and Sawaki, S. (1968). Riemannian manifolds admitting a conformal transformation group. Journal of Differential Geometry, 2: 161-184.

Yano, K. and Kon, M. (1984). Structures on manifolds, World Scientific Publishing Co. Pvt. Ltd., Singapore. 